\title{
Electrocondensation and Evaporation of Attoliter Water Droplets: Direct Visualization Using Atomic Force Microscopy
}

\author{
Narendra Kurra ${ }^{1}$, Adina Scott ${ }^{2}$, and Giridhar U. Kulkarni ${ }^{1}(\bowtie)$ \\ ${ }^{1}$ Chemistry and Physics of Materials Unit and DST Unit on Nanoscience, Jawaharlal Nehru Centre for Advanced Scientific Research, \\ Jakkur PO, Bangalore 560 064, India \\ ${ }^{2}$ Birck Nanotechnology Center, Purdue University, West Lafayette, Indiana 47907, USA \\ Received: 1 February 2010 / Revised: 1 March 2010 / Accepted: 1 March 2010 \\ C The Author(s) 2010. This article is published with open access at Springerlink.com
}

\begin{abstract}
Working with a biased atomic force microscope (AFM) tip in the tapping mode under ambient atmosphere, attoliter $\left(10^{-18} \mathrm{~L}\right)$ water droplet patterns have been generated on a patterned carbonaceous surface. This is essentially electrocondensation of water leading to charged droplets, as evidenced from electrostatic force microscopy measurements. The droplets are unusual in that they exhibit a highly corrugated surface and evaporate rather slowly, taking several tens of minutes.
\end{abstract}

\section{KEYWORDS}

Electrocondensation, attoliter water droplets, biased atomic force microscope (AFM) lithography, electron beam induced deposition, carbonaceous deposition

\section{Introduction}

Water wetting behavior of materials on the nanoscale has attracted a great deal of interest in recent years [1]. Nanoscale patterning using atomic force microscope (AFM)-based surface wetting has emerged in the form of dip-pen nanolithography (DPN) [2]. DPN is a direct write lithographic technique in which an AFM tip is inked with molecules of interest. A narrow gap capillary is formed between the AFM tip and the surface which in the ambient atmosphere leads to the formation of a water bridge, enabling transport of molecules from the tip to the surface. A vast variety of inks made of small organic molecules, colloids, polymers, biological molecules, and metal ions have been patterned onto surfaces of interest with nanoscale resolution via the DPN process [2-4]. Unlike DPN, where water is acting only as a solvent or mediator for molecules, electrochemical DPN utilizes the tiny water meniscus as a nanometer-sized cell in which metal salts can be electrochemically reduced into metals and deposited locally on the surface. Thus, for instance, metallic patterns of $\mathrm{Pt}, \mathrm{Pd}, \mathrm{Cu}, \mathrm{Ag}$, and $\mathrm{Ge}$ have been fabricated using this technique [5]. Besides DPN, there are lithography techniques which rely on the electrolytic decomposition of the water meniscus under a biased AFM tip. Sacha et al. [6] have presented an analytical model to explain the electric field-induced water condensation and bridge formation between an AFM tip and a metal surface under humid conditions. The application of a moderate tip bias of few volts generates an electric field of $10^{8}-10^{10} \mathrm{~V} / \mathrm{m}$ which induces the ionization of water molecules in the meniscus, leading to the generation of oxidative 
species $\left(\cdot \mathrm{OH}, \mathrm{O}^{-}\right)$. These oxidative species produce ionic currents responsible for the local oxidation of the surface, called local anodic oxidation (LAO), which has been employed to produce oxide structures on semiconductors, metals, and other materials [7]. In the case of polymeric films deposited on conductive substrates, the intense fields $\left(10^{8}-10^{9} \mathrm{~V} / \mathrm{m}\right)$ can cause dielectrophoretic manipulation of a thin polymer film softened locally due to Joule heating to its glass transition temperature [8]. Below the characteristic dielectric breakdown voltage of the polymer, mass transport to the tip takes place producing stable protruded patterns [8-10] or features which decay with time [11], while at higher voltages the polymer can become ablated, producing trenches $[8,9,12,13]$.

In most such cases, water does mediate an interaction or a reaction, but needs not be part of the final product itself; its presence is often inferred only from the tip-surface interaction forces [14,15]. Indeed, there are many reports of water bridge formation, experimental as well as theoretical, relating to cantilever dynamics and meniscus forces [16-18]. Water meniscus formation between a tip and a surface has been directly visualized using environmental scanning electron microscopy (SEM) [19, 20]. Only under conditions of high humidity does the AFM tip induce the formation of water droplets on a highly hydrophilic surface (such as mica) [21]. Otherwise, the droplets disappear instantly, and are not amenable to study by current AFM instruments, which typically require several minutes for imaging [22]. Thus, the use of AFM as an effective water dispensing tool is quite limited. We considered that it would be interesting to nucleate and deposit tiny water droplets in a controlled way relying on the electric field from a biased AFM tip. In this article, we demonstrate how a biased AFM tip on a carbonaceous platform is able to induce charged water condensation which could be imaged through its evaporation over several minutes. The carbonaceous platform serves as an insulating layer preserving the charge and minimizing the effects of electrolytic decomposition of water, particularly at high tip voltages. It is also chemically inert and robust. Below, we present our direct observations of the electrocondensation of attoliter $\left(\mathrm{aL}, 10^{-18} \mathrm{~L}\right)$ water droplets and their subsequent evaporation, unusual in many respects.

\section{Experimental}

A n-type silicon wafer $(\rho=4-7 \Omega \cdot \mathrm{cm})$ was cleaned by sonicating in acetone and then rinsing in double distilled water for $2 \mathrm{~min}$. Electron beam induced carbonaceous deposition was performed using a Nova NanoSEM 600 field-emission scanning electron microscope (FESEM) system (FEI Company) in environmental mode (see Fig. 1) making use of the residual hydrocarbons present in the chamber [23]. The pole piece was mounted with a low vacuum detector (LVD) and the chamber was evacuated and subsequently backfilled with water vapor to a pressure of 0.4 Torr. Square shaped carbonaceous deposits $(5 \mu \mathrm{m} \times 5 \mu \mathrm{m})$ were created on the Si substrate at electron beam dosages of $1.3 \mathrm{C} / \mathrm{cm}^{2}$ with an accelerating voltage of $10 \mathrm{kV}$ in the TV mode (area deposition mode) at a working distance of $4 \mathrm{~mm}$. AFM imaging of the carbonaceous deposits as well as bias lithography were carried out at room temperature employing a Dimension 3100 scanning probe microscope (SPM) with NS-IV controller (Veeco, USA) in tapping mode using a Pt-Ir coated Si tip (spring constant $=1-5 \mathrm{~N} / \mathrm{m}$ and resonance frequency $=75 \mathrm{kHz}$ ). The subsequent images were captured in tapping mode AFM using the same tip. Repetitive scanning was done in selected regions using the "Refresh region" command.

\section{Results and discussion}

The thickness of the chosen layer for AFM nanolithography was $\sim 8 \mathrm{~nm}$ as shown by the height profile in Fig. 1(a). AFM lithography was performed on the chosen platform in the tapping mode using a tip voltage of $+10 \mathrm{~V}$ and speed of $1 \mu \mathrm{m} / \mathrm{s}$ with the relative humidity (RH) set at $45 \%$. Following lithographic writing, the topography image showed a pattern, somewhat corresponding to the intended shape "NANO" (Fig. 1(b)). It is remarkable that the raised pattern in some places is as high as $\sim 15 \mathrm{~nm}$ above the carbonaceous platform (see between points $A$ and $B$ in Fig. 1(b)). Lithography performed in the contact mode under similar conditions however, did not produce raised features but instead weak features (around $2 \mathrm{~nm}$, see Fig. S-1 in the Electronic Supplementary Material (ESM)) similar to previous reports on 
carbonaceous films [23]. When the tip was negatively biased, however, the experiment was unsuccessful, whether in contact or tapping mode AFM (see Fig. S-2 in the ESM). Thus, among other conditions, a positively biased tip in tapping mode AFM seems to be important if the high rise features are to be produced. The same tip with a negative bias produced typical LAO patterns on bare regions of the $\mathrm{Si}$ substrate (see between points $C$ and $D$ in Fig. 1(b)), where the height is only $\sim 2.5 \mathrm{~nm}$, as expected [24]. Repeated AFM imaging of the region revealed that the raised structures exhibit a time-dependent behavior (Figs. 1(b)-1(d)). The image in Fig. 1(c) captured after a lapse of 15 min shows the patterned region diminishing in height as depicted in the adjoining profile. Interestingly, there was no trace of the raised pattern after $30 \mathrm{~min}$ as evident from the image in Fig. 1(d); the corresponding height profile closely resembles that of the bare carbonaceous platform (see Fig. 1(a)). The LAO pattern on $\mathrm{Si}$, however, remains unchanged (Fig. 1(d)). From the images in Figs. 1(a) and 1(d), it is clear that the carbonaceous platform itself does not participate chemically, but only witnesses temporary physical changes on the surface leading to the formation of raised features. These raised features, attributed to water condensation from the biased tip, subsequently evaporate, leading to reduced feature heights and their eventual disappearance. As the topography of the surface seen in Fig. 1(d) is very similar to that in Fig. 1(a), one may conclude that the raised features are exclusively formed by water with no visible contamination from the carbonaceous species. The pattern, being made of water, has low aspect ratio with blurred boundaries. It appears that this is an electrocondensation process induced by a biased AFM tip under moderately humid conditions (with $\mathrm{RH}$ of $45 \%$ ). It works at lower humidity values as well but, interestingly, causes uncontrolled water condensation beyond $\mathrm{RH}$ of $55 \%$ (see Fig. S-3 in the ESM). To our knowledge, such raised patterns of electrocondensed water and their disappearance with time have not been observed hitherto.

The lithography process was carried out at different tip voltages and velocities (Fig. 2). Figure 2(a) shows a typical image scanned within the first minute of drawing lines of water condensates at tip voltages of
5,7 , and $9 \mathrm{~V}$ (tip velocity $=1 \mu \mathrm{m} / \mathrm{s}$ ); increasing tip bias produced increasingly prominent water condensates. Below $3 \mathrm{~V}$, no condensation was observed. The $z$-profiles showed a linear increase in the height of the condensate with increasing tip bias but beyond 8 $\mathrm{V}$, the height decreased (Fig. 2(b)). This trend is also reflected in the volume. The volume of water calculated using SPIP software [25], increases gradually up to $8 \mathrm{~V}$ (see Fig. 2(d)) and seems remain almost constant thereafter, at around $\sim 20 \mathrm{aL}$. Higher voltages may cause increased ionization of water molecules leading to ionic currents [26]. The scatter in the volume data in Fig. 2(d) is therefore not surprising. The influence of tip velocity on the electrocondensation process is relatively small (Figs. 2(c) and 2(e)). The right-hand images in Fig. 2(a) corresponding to velocities of 0.1 and $1.0 \mu \mathrm{m} / \mathrm{s}$ (tip voltage $=10 \mathrm{~V}$ ) show only marginal differences. The height variation is between $20-27 \mathrm{~nm}$, with a change in trend around $1.0 \mu \mathrm{m} / \mathrm{s}$. Greater dwell time at lower speeds can make the tip dump more water on the surface, while for velocities above $2 \mu \mathrm{m} / \mathrm{s}$, the water meniscus itself may become unstable. Such situations are quite common while performing DPN [4]. As regards water volume (Fig. 2(e)), it is difficult to decipher a definite trend due to data scatter, although a gradual decrease is evident. At lower tip velocities or smaller areas, one may also expect effects due to ionization and Joule heating (see Fig. S-4 in the ESM).

Electrostatic force microscopy (EFM) was carried out on a water condensate at a tip lift height of $120 \mathrm{~nm}$ (Fig. 3). The phase image obtained with a tip bias of $+2 \mathrm{~V}$ (Fig. 3(a)) shows a bright contrast corresponding to the water feature, whereas that in Fig. 3(b) recorded with a tip bias of $-2 \mathrm{~V}$ showed a darker contrast. This color contrast relates to charge accumulation in the water condensate. A brighter contrast from the positive bias data is taken to indicate positive charge accumulation in the condensate [27]. Figures 3(c) and 3(d) show diminishing features due to water evaporation. The charge seems to dissipate locally as the molecules evaporate. Although this observation is qualitative, it certainly indicates the presence of electrical charges on the condensed water features from the lithography process. Charge quantification was not attempted due to the temporal nature of the features. 

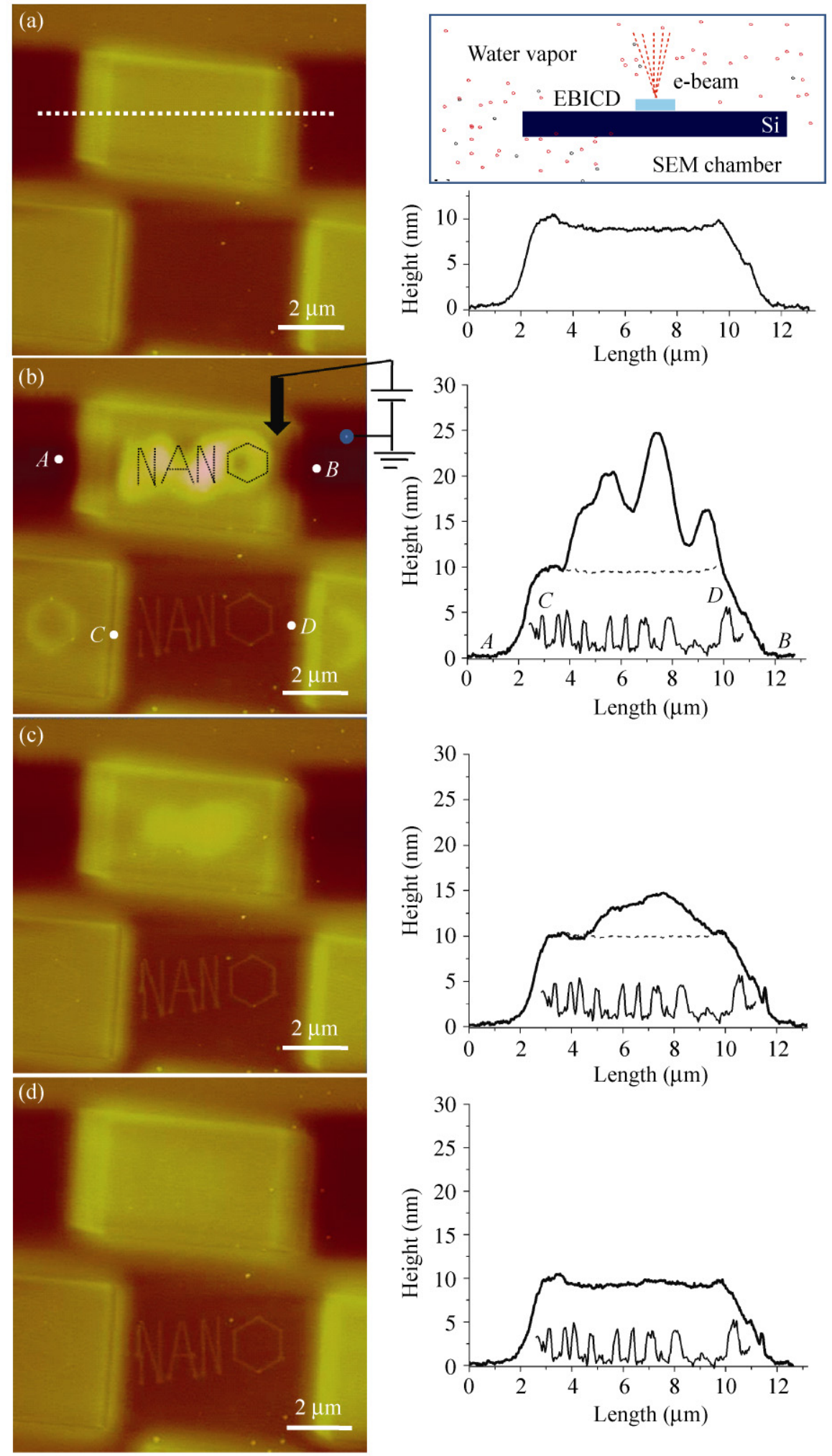

Figure 1 AFM images (left) and corresponding height profiles (right) illustrating the electrocondensation process. The carbonaceous platforms on a Si substrate are shown in (a). The process of electron beam (e-beam) induced deposition (EBICD) is shown schematically in the inset. (b) Shows the same region immediately after writing a water pattern ("NANO") on a carbonaceous platform with a positively-biased AFM tip (between points $A$ and $B$ ). For comparison, "NANO" is also written using a standard local anodic oxidation (LAO) process employing a negatively-biased tip (between points $C$ and $D$ ). Over time, the water patterns dissipate, as shown in (c) and (d), whereas the LAO pattern remains unchanged 

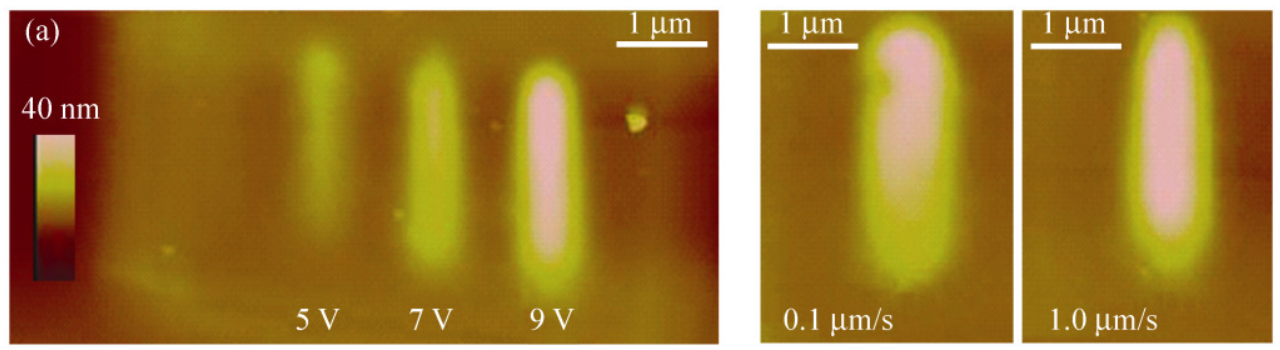

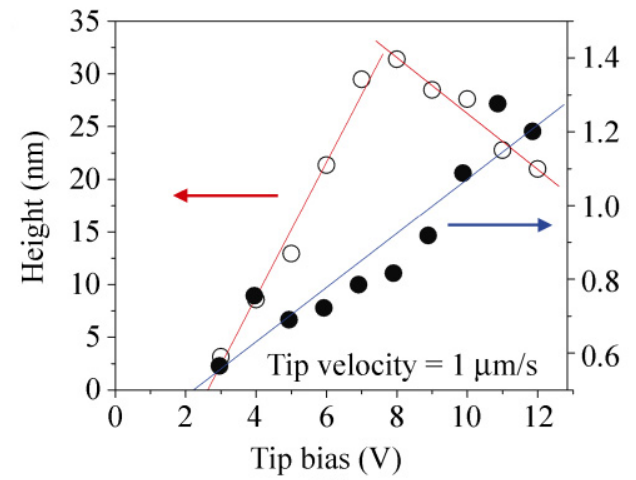

(b)

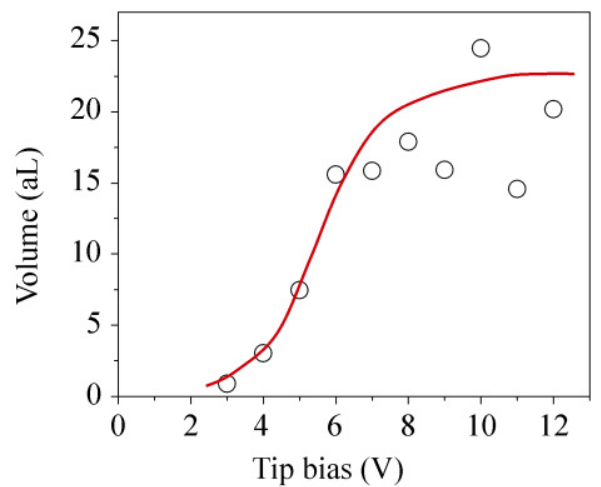

(d)

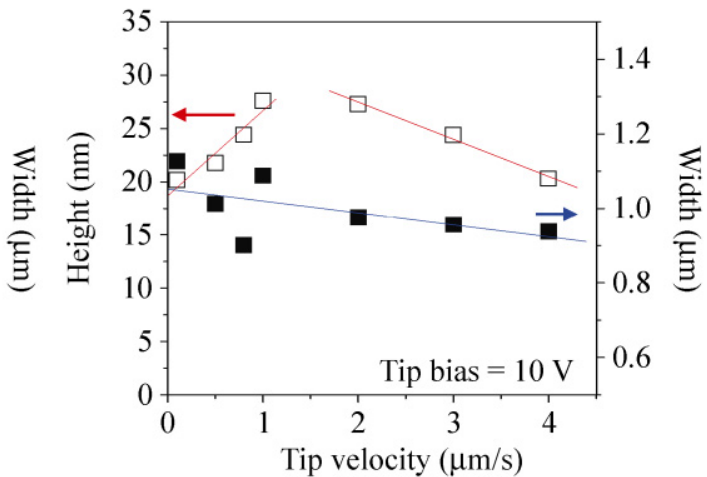

(c)

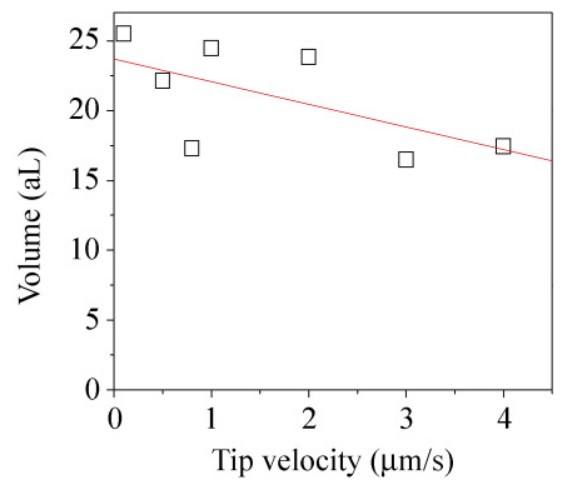

(e)

Figure 2 The dimensions of the water pattern can be controlled by varying the writing conditions. The effects of tip bias (left) and tip velocity (right) during patterning were characterized. AFM images of representative $2 \mu \mathrm{m}$ single-pass lines written under different conditions are shown in (a). The variations in water feature height and width with respect to tip bias and velocity are shown in (b) and (c), respectively. Similarly, the variations in volume are shown in (d) and (e), respectively

It appears that many experimental parameters are involved in water condensation. In the tapping mode AFM with the given parameters, the tip makes only intermittent contact with the surface and therefore, the water bridge formation is not spontaneous. A certain threshold voltage $\left(V_{\mathrm{Th}} \sim+3 \mathrm{~V}\right.$ in this case) is therefore required to induce the formation of a water bridge $[16,17]$. The substrate also seems to play an important role. The carbonaceous species is dielectric [28], but its surface functionality is locally polarizable, a property which owes much to the presence of moisture during deposition using an electron beam.
During the AFM lithography process, the deposited water carries positive charges from the positively biased tip (negative bias leads to no water condensation) which means that the surface of the carbonaceous layer is polarisable only with positive charges. Further, the contact mode would not be appropriate for water condensation under these conditions. Higher voltage conditions are also not favorable as ionic currents would destabilize the condensed water droplets. This is more so with negative voltages [11], adding another reason to why negative tip voltages could not induce electrocondensation in our studies. 


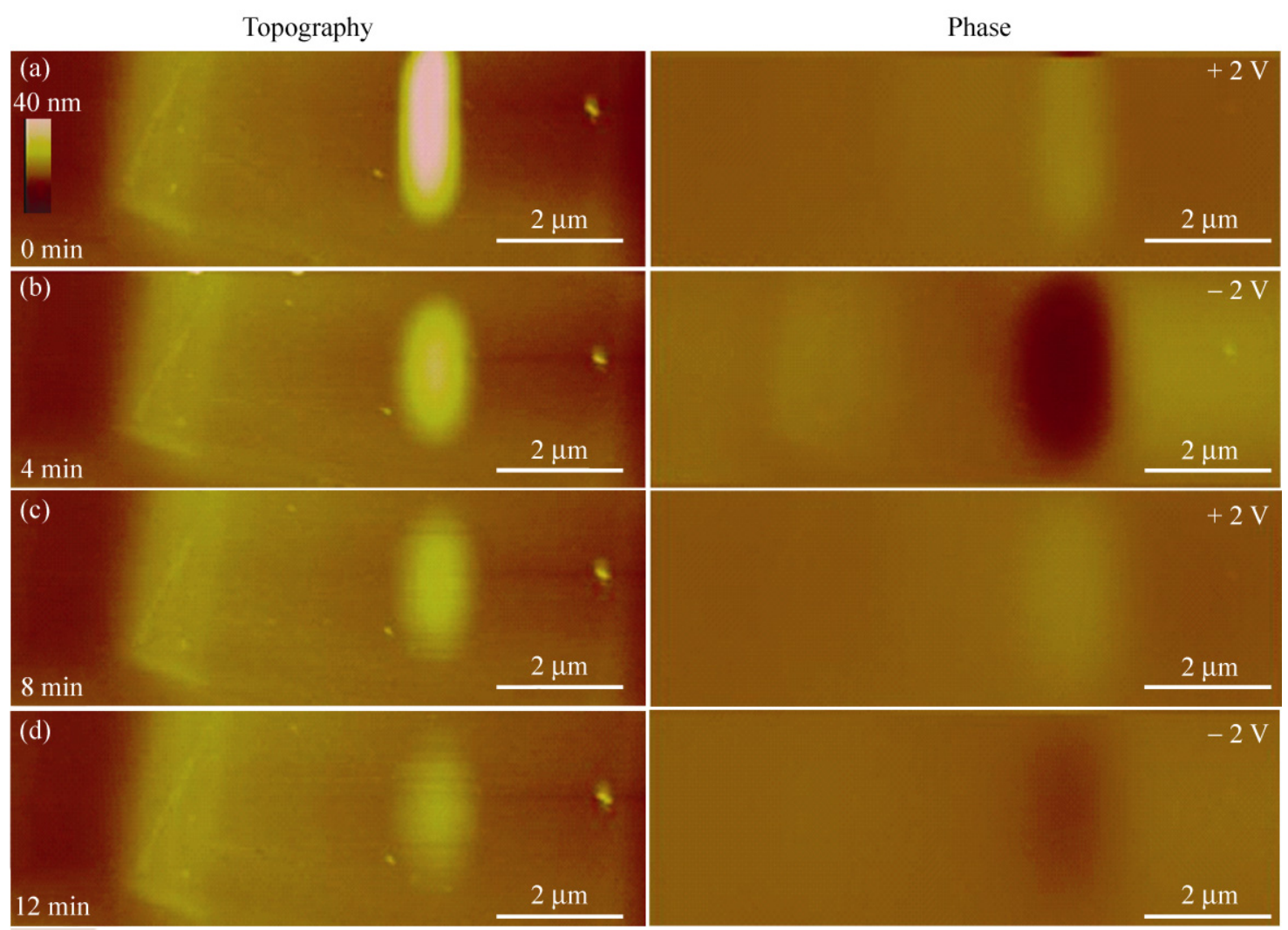

Figure 3 Electrostatic force microscopy (EFM) was used to probe the charge and polarization within the water droplet. Topography images (left) and corresponding EFM phase images of a water feature as it evaporates are shown. Images (a) and (c) were taken with positive EFM tip voltage $(+2 \mathrm{~V})$ whereas $(b)$ and $(d)$ where taken with negative EFM voltage $(-2 \mathrm{~V})$. The reversal of contrast for different tip biases in EFM clearly indicates that the water droplet is charged. The local charge dissipates as the water evaporates

Having realized positive charge accumulation in electrocondensed water on a carbonaceous platform, we studied its interaction with a negatively biased tip (Fig. 4). For this purpose, a large water drop was created on a carbonaceous platform as shown in Fig. 4(a), by drawing closely spaced lines with a tip bias of $+10 \mathrm{~V}$. Although the topography is not quite even (discussed later), it enabled us to make further observations. The image in Fig. 4(b) obtained after patterning on the water feature with a $-10 \mathrm{~V}$ biased tip, shows that water can be instantaneously evaporated locally. Although the intended pattern, " $\mathrm{K}$ " is not seen distinctly, water evaporation in the region is quite apparent. It appears that local neutralization of the positive charge by the negatively biased tip leads to an ionic current and Joule heating causing instantaneous evaporation. This is also reflected in terms of contrast in the phase images (Fig. 4(b)).
From the above observations (Figs. 1-3), it is clear that a positively biased AFM tip can trigger electrocondensation. The implications of surface charge on the evaporation of droplets are striking. The time-dependent behavior of the water condensate is shown in Fig. 5 in terms of image contrast, z-profiles, and volume. The water condensate was created with a tip bias of $+10 \mathrm{~V}$, indicated as $0 \mathrm{~min}$ in Fig. 5(a). The gradual evaporation of the water condensate is observed through the images obtained after 8 and 16 min with complete evaporation after $24 \mathrm{~min}$. Correspondingly, the height of the water condensate decreases from 25 to $2 \mathrm{~nm}$ as shown by AFM z-profiles in Fig. 5(b). There was also considerable smearing as seen from the width variation, $\sim 0.5$ to $2.5 \mu \mathrm{m}$. Figures. 5(c) and 5(d) show the trends in height and width variations, respectively, for water condensates produced with different tip voltages. A gradual variation in height is 


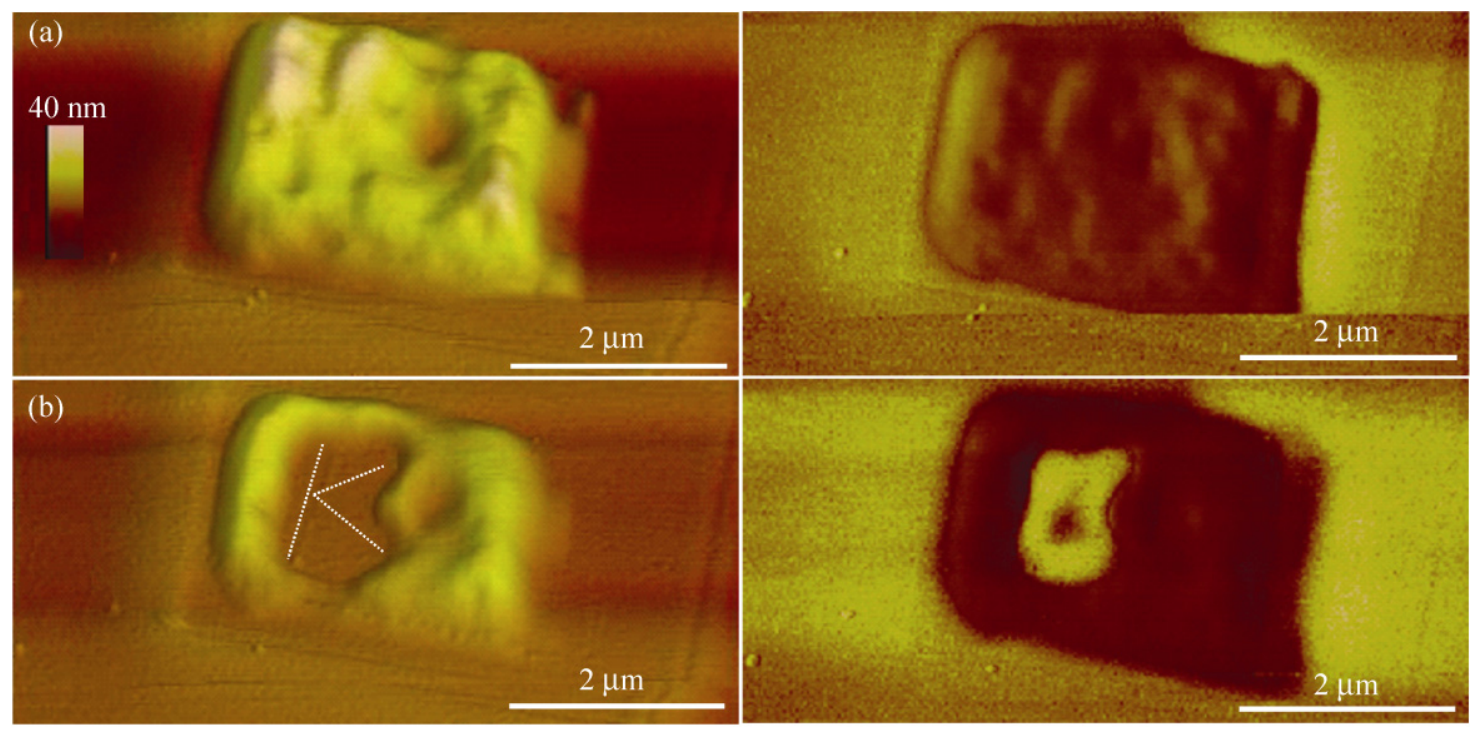

Figure 4 Subtractive water patterning by tip-induced evaporation. (a) A large water feature obtained by drawing closely spaced lines using $+10 \mathrm{~V}$ tip bias. By applying negative bias $(-10 \mathrm{~V})$, it is possible to induce rapid evaporation, as shown in (b). The dotted line shows schematically the nominal feature (the letter "K"). The corresponding phase images of (a) and (b) are shown on the right-hand side

observed, where the different curves are seen to be raised depending on the initial height (Fig. 5(c)). The $8 \mathrm{~V}$ curve is on the top extending up to $38 \mathrm{~min}$. On the other hand, the width variation is not found to be monotonic. The width increases in each case up to a certain point, beyond which it decreases; the turnaround time itself is found to increase with the tip voltage employed to create the water condensate. Interestingly, the low bias curves $(4,5,6$, and $8 \mathrm{~V})$ group themselves with an initial width at $\sim 0.5 \mu \mathrm{m}$. The $10 \mathrm{~V}$ and $12 \mathrm{~V}$ curves are much wider (see Fig. 5(d)). Similar trends are observed in the volume data (Fig. 5(e)). We see that the water condensates created using lower tip biases evaporate rather quickly (within $\sim 12 \mathrm{~min}$ ), while those obtained using a bias above $8 \mathrm{~V}$, exhibit an initial drop lasting $5 \mathrm{~min}$ and a more gradual evaporation during the following $20 \mathrm{~min}$ or so. The behavior with the $8 \mathrm{~V}$ curve is quite unique, with an overall gradual evaporation going beyond $25 \mathrm{~min}$. The maximum evaporation rate is estimated to be $2 \mathrm{aL} / \mathrm{min}$ (see Fig. S-5 in the ESM).

The evaporation of macro- or micro-droplets of water is known to be a rapid process; the evaporation rate of a water droplet on a poly(methylmethacrylate) (PMMA) surface under similar humidity conditions is typically $0.1 \mu \mathrm{L} / \mathrm{min}$ [29]. Assuming that the observed trends in evaporation are independent of the droplet size, attoliter volumes of water such as those shown in Fig. 4(a), should evaporate within picoseconds $\left(10^{-12} \mathrm{~s}\right)$. In contrast, we observe that attoliter quantities of water evaporate very slowly, indicating that quite different physical mechanisms apply to nanoscale volumes $[30,31]$. Besides, the droplets of water are highly charged and the dimensions are perhaps small relative to the screening lengths in water, which are typically of the order of a few hundred nanometers [32]. Since the carbonaceous platform serving as the substrate for water is dielectric, the dissipation of charges is expected to be slow. It appears that water molecules require extraneous energy to leave the droplet surface $[30,31,33,34]$. As a result, the rate of evaporation is significantly reduced, enabling us to visualize the droplets on a macroscopic timescale. Interestingly, the evaporation curves in Fig. 5(e) show that when the droplet volume is above $20 \mathrm{aL}$ (see the 10 and $12 \mathrm{~V}$ curves in Fig. 5(e)), initial evaporation is fast ( $\sim 5 \mathrm{~min})$ and thereafter becomes gradual, much the same way as microliter droplets would behave [29]. Smaller droplets $(<20 \mathrm{aL}$, see the $8,6,5,4$, and $3 \mathrm{~V}$ curves in Fig. 5(e)), differ from this behavior in that their trends are relatively monotonic.

The electrocondensed water on the carbonaceous 

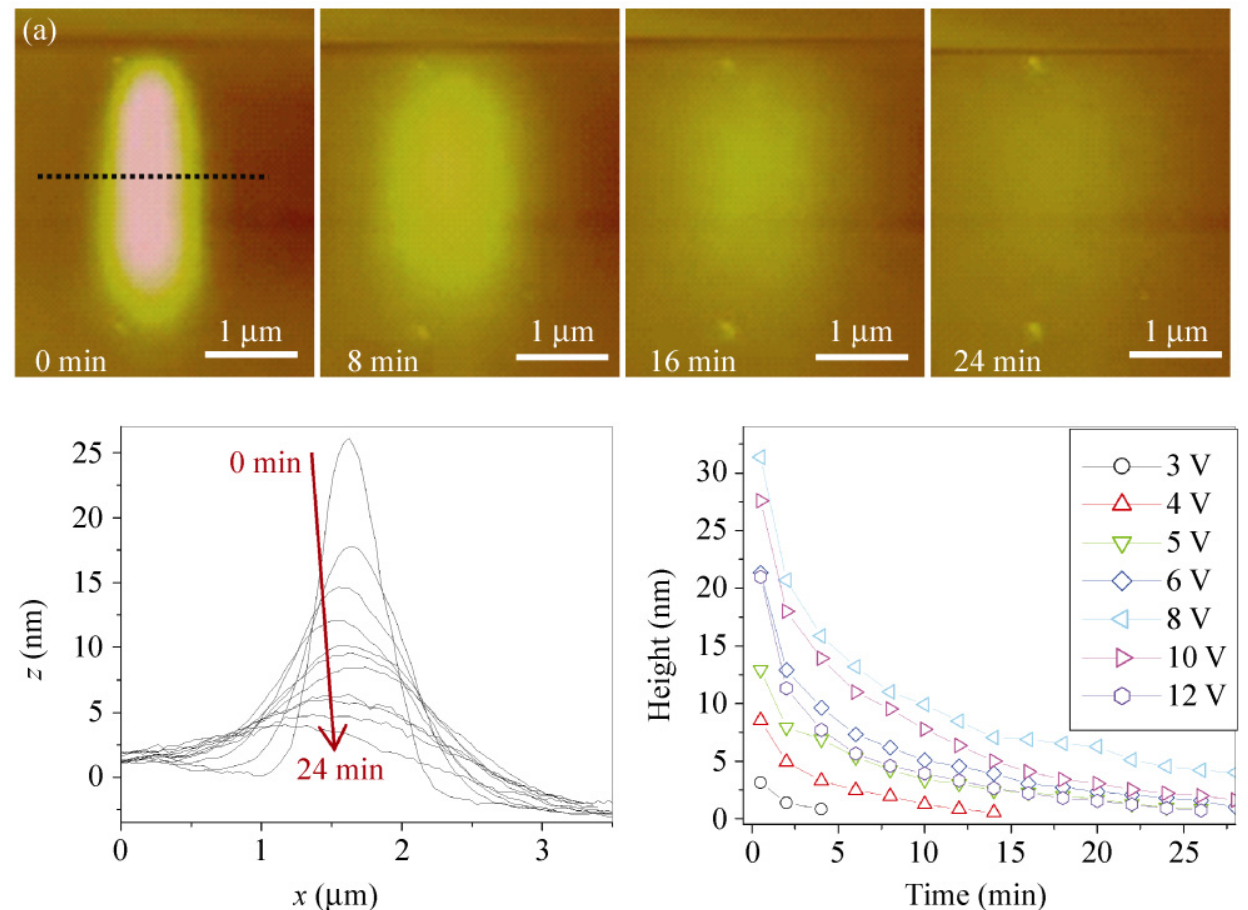

(b)

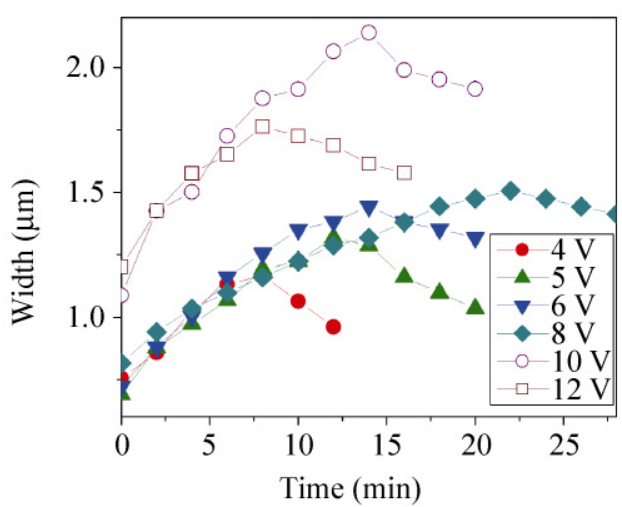

(d)

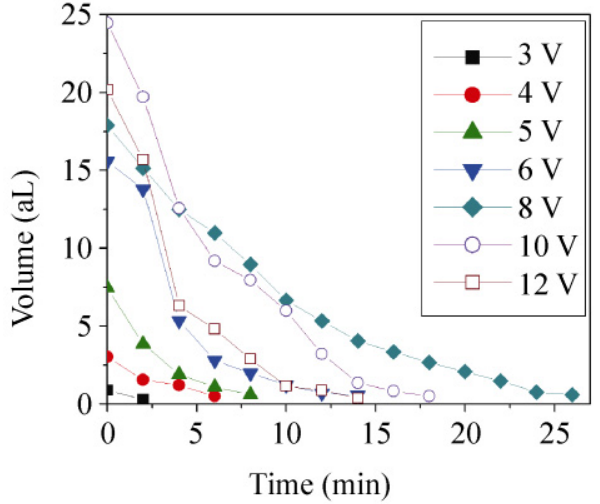

(e)

Figure 5 Evolution of water feature size and shape during evaporation. A representative series of images taken at different time after writing are shown in (a). (b) Height profiles of an evaporating drop illustrating that the height decreases and the width increases over a period of $24 \mathrm{~min}$. Height variation (c), width variation (d), and volume variation (e) of water condensates at different tip voltages

platform is different from standard droplets in yet another sense. One may recall the topography of the image shown in Fig. 4(a), where the square water island is seen to have a highly uneven surface, a behavior distinctly different from that of bulk water. Another example is the topography image in Fig. 6(a) of a condensate of a ring of water, which did not show any tendency to collapse into a rounded droplet (also see Fig. S-6 in the ESM for time-dependent behavior). The overlapping $z$-profile shows unusually sharp local morphology and corrugations of the water condensate. This is illustrated schematically in Fig. 6(b). Small volumes of water droplets combined with localized charges arising from alignment of water dipoles under the electric field may result in special properties different from the macroscopic behavior of water condensates [31]. The detailed mechanism of electrocondensation and evaporation kinetics needs further study. 


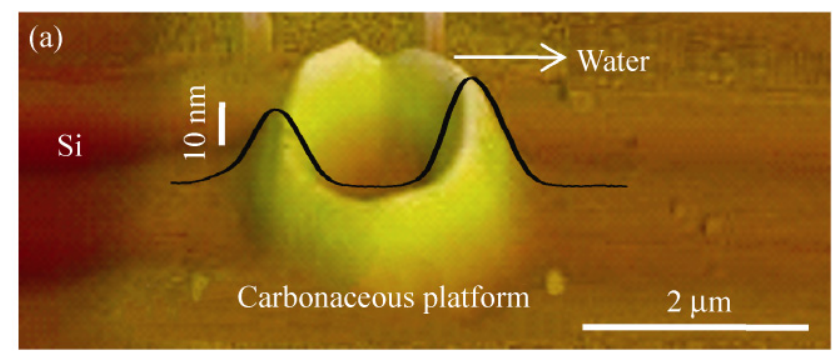

(b)
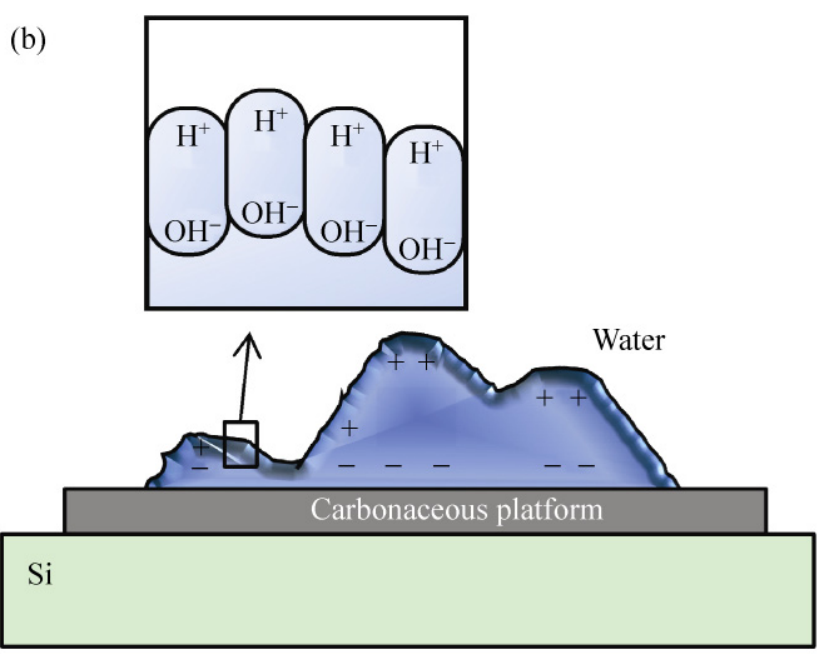

Figure 6 (a) AFM topography image and corresponding line profile of a hexagonal water pattern showing the unusually sharp local water morphology that can be achieved using AFM-induced electrocondensation. The maximum height of the water feature is $27 \mathrm{~nm}$. The schematic drawing in (b) illustrates the presence of local tip-induced charge and polarization in the water which contributes to the observed anomalous droplet shapes and evaporation characteristics

\section{Conclusions}

We have arrived at a set of conditions for the electrocondensation of water from the ambient on a dielectric carbonaceous surface using a biased AFM tip. The carbonaceous regions on Si were created by electron beam induced deposition using residual hydrocarbons along with water vapor at 0.4 Torr. Working in tapping mode AFM, positive tip voltages above $3 \mathrm{~V}$ (preferably below $9 \mathrm{~V}$ ) and velocities up to $4 \mu \mathrm{m} / \mathrm{s}$ were found to be optimal for electrocondensation of water droplets. The volume of the water condensates was typically $10-30 \mathrm{aL}$, and they carried a positive surface charge. Interestingly, the surface of the condensates was highly corrugated. We found that the evaporation rates were unusually low $(\sim 2 \mathrm{aL} / \mathrm{min})$, enabling direct observation using AFM, hitherto considered not feasible. The carbonaceous surface however, was left unaltered during the process. Our study provides the first evidence of electrocondensation and evaporation behavior of attoliter quantities of water.

\section{Acknowledgements}

The authors thank Professor C. N. R. Rao, Fellow of Royal Society (FRS) for his encouragement. Support from the Department of Science and Technology, Government of India is gratefully acknowledged. N. K. acknowledges Council of Scientific and Industrial Research (CSIR) for funding. N. K. acknowledges Ritu for reading the manuscript. The authors thank Veeco India Nano-technology Laboratory at Jawaharlal Nehru Centre for Advanced Scientific Research (JNCASR) for the AFM facility. A. S. acknowledges INDO-US Science $\&$ Technology Forum (IUSSTF) for funding.

Electronic Supplementary Material: AFM images showing that biased AFM nanolithography on carbonaceous platforms gives either condensation of water or chemical modification depending on the mode of tip operation (tapping or contact), polarity of the applied bias, the tip velocity and the relative humidity, plots of the evaporation rates of attoliter water condensates and a series of AFM images illustrating the evaporation of a ring of water condensate over time are available in the online version of this article at http://dx.doi.org/10.1007/s12274-010-1034-0 and are accessible free of charge.

Open Access: This article is distributed under the terms of the Creative Commons Attribution Noncommercial License which permits any noncommercial use, distribution, and reproduction in any medium, provided the original author(s) and source are credited.

\section{References}

[1] Verdaguer, A.; Sacha, G. M.; Bluhm, H. Salmeron, M. Molecular structure of water at interfaces: Wetting at the nanometer scale. Chem. Rev. 2006, 106, 1478-1510.

[2] Salaita, K.; Wang, Y. H.; Mirkin, C. A. Applications of dip-pen nanolithography. Nat. Nanotechnol. 2007, 2, 145-155.

[3] Ginger, D. S.; Zhang, H.; Mirkin, C. A. The evolution of 
dip-pen nanolithography. Angew. Chem. Int. Ed. 2004, 43, 30-45.

[4] Piner, R. D.; Zhu, J.; Xu, F.; Hong, S.; Mirkin, C. A. "Dip-pen" nanolithography. Science 1999, 283, 661-663.

[5] Li, Y.; Maynor, B. W.; Liu, J. Electrochemical AFM “dip-pen" nanolithography. J. Am. Chem. Soc. 2001, 123, 2105-2106.

[6] Sacha, G. M.; Verdaguer, A.; Salmeron, M. Induced water condensation and bridge formation by electric fields in atomic force microscopy. J. Phys. Chem. B 2006, 110, 14870-14873.

[7] Xie, X. N.; Chung, H. J.; Sow, C. H.; Wee, A. T. S. Nanoscale materials patterning and engineering by atomic force microscopy nanolithography. Mat. Sci. Eng. R: Rep. 2006, 54, 1-48.

[8] Lyuksyutov, S. F.; Vaia, R. A.; Paramonov, P. B.; Juhl, S.; Waterhouse, L.; Ralich, R. M.; Sigalov, G.; Sancaktar, E. Electrostatic nanolithography in polymers using atomic force microscopy. Nat. Mater. 2003, 2, 468-472.

[9] Lyuksyutov, S. F.; Paramonov, P. B.; Sharipov, R. A.; Sigalov, G. Induced nanoscale deformations in polymers using atomic force microscopy. Phys. Rev. B 2004, 70, 174110.

[10] Juhl, S.; Phillips, D.; Vaia, R. A.; Lyuksyutov, S. F.; Paramonov, P. B. Precise formation of nanoscopic dots on polystyrene film using $z$-lift electrostatic lithography. Appl. Phys. Lett. 2004, 85, 3836-3838.

[11] Reagan, M. A.; Kashyn, D.; Juhl, S.; Vaia, R. A.; Lyuksyutov, S. F. Electric charging and nanostructure formation in polymeric films using combined amplitude-modulated atomic force microscopy-assisted electrostatic nanolithography and electric force microscopy. Appl. Phys. Lett. 2008, 93, 033109.

[12] Martín, C.; Rius, G.; Borrisé, X.; Pérez-Murano, F. Nanolithography on thin layers of PMMA using atomic force microscopy. Nanotechnology 2005, 16, 1016-1022.

[13] Vijaykumar, T.; Kulkarni, G. U. Electrostatic nanolithography on PVP films for patterning metal nanocrystals and fullerenes. Nanotechnology 2007, 18, 445303 .

[14] Jang, J.; Schatz, G. C.; Ratner, M. A. Capillary force on a nanoscale tip in dip-pen nanolithography. Phys. Rev. Lett. 2003, 90, 156104.

[15] Stifter, T.; Marti, O.; Bhushan, B. Theoretical investigation of the distance dependence of capillary and van der Waals forces in scanning force microscopy. Phys. Rev. B 2000, 62, 13667-13673.

[16] Calleja, M.; Tello, M.; García, R. Size determination of field-induced water menisci in noncontact atomic force microscopy. J. Appl. Phys. 2002, 92, 5539-5542.

[17] Gómez-Moñivas, S.; Sáenz, J. J.; Calleja, M.; García, R. Field-induced formation of nanometer-sized water bridges. Phys. Rev. Lett. 2003, 91, 056101.

[18] Cramer, T.; Zerbetto, F.; García, R. Molecular mechanism of water bridge buildup: Field-induced formation of nanoscale menisci. Langmuir 2008, 24, 6116-6120.

[19] Schenk, M.; Füting, M.; Reichelt, R. Direct visualization of the dynamic behavior of a water meniscus by scanning electron microscopy. J. Appl. Phys. 1998, 84, 4880-4884.

[20] Weeks, B. L.; Vaughn, M. W.; DeYoreo, J. J. Direct imaging of meniscus formation in atomic force microscopy using environmental scanning electron microscopy. Langmuir 2005, 21, 8096-8098.

[21] Piner, R. D.; Mirkin, C. A. Effect of water on lateral force microscopy in air. Langmuir 1997, 13, 6864-6868.

[22] Méndez-Vilas, A.; Jódar-Reyes, A. B.; González-Martín, M. L. Ultrasmall liquid droplets on solid surfaces: Production, imaging, and relevance for current wetting research. Small 2009, 5, 1366-1390.

[23] Avramescu, A.; Ueta, A.; Uesugi, K.; Suemune, I. Atomic force microscope lithography on carbonaceous films deposited by electron-beam irradiation. Appl. Phys. Lett. 1998, 72, 716-718.

[24] Pérez-Murano, F.; Abadal, G.; Barniol, N.; Aymerich, X.; Servat, J.; Gorostiza, P.; Sanz, F. Nanometer-scale oxidation of $\mathrm{Si}(100)$ surfaces by tapping mode atomic force microscopy. J. Appl. Phys. 1995, 78, 6797-6801.

[25] Note: Volumes of water condensates are calculated using SPIP software (www.imagemet.com)

[26] Pinkerton, T. D.; Scovell, D. L.; Johnson, A. L.; Xia, B.; Medvedev, V.; Stuve, E. M. Electric field effects in ionization of water-ice layers on platinum. Langmuir 1999, 15, 851-856.

[27] Scanning Probe Microscopy and Spectroscopy: Theory, techniques, and applications; Bonnell, D. A., Ed.; Wiley-VCH: New York, 2001.

[28] Miura, N.; Ishii, H.; Shirakashi, J. I.; Yamada, A.; Konagai, M. Electron-beam-induced deposition of carbonaceous micro structures using scanning electron microscopy. Appl. Surf. Sci. 1997, 113-114, 269-273.

[29] Kim, J. H.; Ahn, S. I.; Kim, J. H.; Zin, W. C. Evaporation of water droplets on polymer surfaces. Langmuir 2007, 23, 6163-6169.

[30] Hock, C.; Schmidt, M.; Kuhnen, R.; Bartels, C.; Ma, L.; Haberland, H.; v. Issendorff, B. Calorimetric observation of the melting of free water nanoparticles at cryogenic temperatures. Phys. Rev. Lett. 2009, 103, 073401.

[31] Znamenskiy, V.; Marginean, I.; Vertes, A. Solvated ion evaporation from charged water nanodroplets. J. Phys. Chem. A 2003, 107, 7406-7412.

[32] Daub, C. D.; Bratko, D.; Leung, K.; Luzar, A. Electrowetting at the nanoscale. J. Phys. Chem. C 2007, 111, 505-509.

[33] Iribarne, J. V.; Thomson, B. A. On the evaporation of small ions from charged droplets. J. Chem. Phys. 1976, 64, 2287-2294.

[34] Krymskiǐ, G. F.; Pavlov, G. S. Electrostatic model of water vapor condensation. Dokl. Phys. 2008, 53, 310-311. 